\title{
Linking behavioral and neurophysiological indicators of perceptual tuning to language
}

\author{
Eswen Fava ${ }^{1}$, Rachel Hull ${ }^{1}$ and Heather Bortfeld ${ }^{2,3}$ * \\ Department of Psychology, Texas A\&M University, College Station, TX, USA \\ 2 Department of Psychology, University of Connecticut, Storrs, CT, USA \\ ${ }^{3}$ Child Lanquage Studies, Haskins Laboratories, New Haven, CT, USA
}

\section{Edited by:}

Judit Gervain, CNRS - Université Paris

Descartes, France

\section{Reviewed by:}

Judit Gervain, CNRS - Université Paris Descartes, France

Krista Byers-Heinlein, Concordia

University, Canada

*Correspondence:

Heather Bortfeld, Department of

Psychology, University of Connecticut,

Storrs, CT 06269-1020, USA.

e-mail: heather.bortfeld@uconn.edu
Little is known about the neural mechanisms that underlie tuning to the native language(s) in early infancy. Here we review language tuning through the lens of type and amount of language experience and introduce a new manner in which to conceptualize the phenomenon of language tuning: the relative speed of tuning hypothesis. This hypothesis has as its goal a characterization of the unique time course of the tuning process, given the different components (e.g., phonology, prosody, syntax, semantics) of one or more languages as they become available to infants, and biologically based maturational constraints. In this review, we first examine the established behavioral findings and integrate more recent neurophysiological data on neonatal development, which together demonstrate evidence of early language tuning given differential language exposure even in utero. Next, we examine traditional accounts of sensitive and critical periods to determine how these constructs complement current data on the neural mechanisms underlying language tuning. We then synthesize the extant infant behavioral and neurophysiological data on monolingual, bilingual, and sensory deprived tuning, thereby scrutinizing the effect of these three different language profiles on the specific timing, progression, and outcome of language tuning. Finally, we discuss future directions researchers might pursue to further understand this aspect of language development, advocating our relative speed of tuning hypothesis as a useful framework for conceptualizing the complex process by which language experience works together with biological constraints to shape language development.

Keywords: near-infrared spectroscopy, perceptual tuning, language development, sensory deprivation, monolingual/ bilingual

\section{INTRODUCTION}

Infants tune to the specific language(s) in their environment very quickly. Much evidence suggests that an individual infant's early language exposure is critical to this tuning process, but we know relatively little about the underlying neural mechanisms that facilitate it. For example, do neural pathways that support tuning to a single language differ from those that support tuning to two (or more) languages? If the timing of language exposure matters, does early tuning to a single native language limit the neural mechanisms available for later acquisition of languages? This review examines data from behavioral and neurophysiological research, following the developmental timeline, to examine how biological maturation interacts with language experience/exposure to influence the underlying neural mechanisms that support language tuning.

In what follows, we consider when experience with language truly begins, and how that experience impacts the development of neural pathways in support of language learning. We then review classic and more recent research on neonates, young infants, and older infants to better understand what is known (and unknown) about the different stages of postnatal language learning. And, since substantial evidence indicates that language learning is influenced by an infant's particular language profile (i.e., single versus multiple language experience), we also explore how perceptual tuning specifically to language (i.e., "language tuning") fits into the oft debated concept of critical (or sensitive) periods. Finally, we explore how data collected using neurophysiological methodologies can add to what is currently known about the processes of infant perceptual tuning, in general, and language development, in particular. Based on our synthesis of these diverse sources of data, we conclude with the introduction of a new manner in which to conceptualize language tuning: the relative speed of tuning hypothesis.

\section{EARLY EXPERIENCE AND ITS INFLUENCE ON NEURAL AND BEHAVIORAL CORRELATES OF LANGUAGE TUNING}

Strict interpretations of language development as completely biologically endowed or completely experience-based have loosened in recent years. Although researchers accept that the biological basis for learning language is in place and at work at birth, there is also substantial appreciation for the fact that changes in environment have profound effects on language outcome. Indeed, there is considerable evidence that critical interactions between neural biases and environmental shaping are at work in utero.

For example, external sound stimuli are "heard" through tissue and liquid barriers of the womb, which excludes frequencies greater than $5000 \mathrm{~Hz}$ (Jardri et al., 2008). In spite of sound filtration by the womb, numerous researchers have used behavioral and physiological measures - such as heart rate and movement - to extrapolate information about when auditory processing begins in utero and what sorts of distinctions the developing fetus can make at various points in gestational time. Lecanuet et al. (1995) provided some of 
the first physiological (heart rate) data suggesting that fetal hearing occurs before 28 gestational weeks. In fact, the fetus responds to sound at 22 gestational weeks (Hepper and Shabidullah, 1994) and habituates to repeated sound stimuli at 32 gestational weeks (Morokuma et al., 2004). Moreover, as babies near term, their sensitivity to more complex auditory stimuli improves, allowing them to perceive variations in music (Kisilevsky et al., 2004) and differentiate between prosodic cues in familiar and novel rhymes (Decasper et al., 1994).Although these studies illustrate a suprasegmental level of language exposure and processing, our concept of true language "experience" should not begin only at birth, as other aspects of language are likewise capable of passing through the uterine wall. This implies a currently unknown threshold in prenatal auditory processing. To better understand the true nature of the intrauterine experience of speech, further research is needed.

The domain of fetal development highlights how biology and environment have already combined prenatally to set the process of language learning in motion. Our position is that the development of neural pathways supporting language begins in utero, and is shaped by the interaction of biology and language experience even then. Understanding the extent and implication of that interaction will require data, both behavioral and neurophysiological, from multiple sources. However, in order to begin to examine language tuning, we must first understand to which aspects of language infants are initially sensitive. Fortunately, we have a wealth of data that can inform us about the neural mechanisms already available to support language development once a baby is born.

\section{BEHAVIORAL AND NEUROPHYSIOLOGICAL EVIDENCE OF NATIVE/NON-NATIVE SPEECH PERCEPTION IN NEONATES}

The most compelling initial evidence about the precocity of infants' language abilities came from a series of behavioral studies showing that neonates are capable of performing various cross-language discriminations (Mehler et al., 1978, 1988; Moon et al., 1993; Nazzi et al., 1998; Ramus et al., 2000). Specifically, these studies revealed that neonates prefer their native language over another language with a dissimilar rhythmic structure. Furthermore, this preference - for the prosodic pattern of the native tongue - has been shown to stem from prenatal experience with native speech (Moon et al., 1993). The preference remains constant as well, including when babies are exposed to two languages (English and Tagalog) in utero. In the case of two languages, infants display equal preference for each when tested as newborns (Byers-Heinlein et al., 2010). Moreover, the data show that Chinese-English bilingually exposed neonates showed intermediate patterns of preference for English compared with Tagalog, but importantly, these newborns are able to discriminate English from Tagalog. Thus, given prenatal experience with more than one language, neonates appear capable of discriminating between the two.

Neonatal near-infrared spectroscopy (NIRS) studies have added support to these behavioral findings, demonstrating distinct hemodynamic response patterns given different syntactic structures (Gervain et al., 2008). Specifically, Gervain et al. (2008) observed increased hemodynamic activity to a repeated syllable sequence in both temporal and left frontal regions in contrast to an unchanging hemodynamic response to control sequences. Differences between the two auditory conditions were observed in the first few trials of the study, as well as across the course of the experiment. The increased hemodynamic response to the repetition sequence in comparison with the control sequences suggests that neonates possess a (perhaps automated) neural mechanism responsible for detecting repetitions. Furthermore, a familiarity effect was inferred by the researchers from the increased hemodynamic response observed during later trials in response to repetition and not to control sequences. As the authors concluded, these data may demonstrate an early neural sensitivity to configurations of auditory stimuli that are often heard in speech.

Near-infrared spectroscopy has also been used to test neonates between 2 and 5 days of age as they listened to recorded speech samples in their native language. Peña et al. (2003) found that native language processing elicited focal regions of activation, including in the dorsolateral prefrontal cortex, the primary and auditory association cortices, and the supramarginal gyrus (a portion of Wernicke's area). Results from this study demonstrated that monolingual neonates already show increased left relative to right temporal activation in response to forward relative to reversed speech. More recently, Saito et al. (2007) used NIRS to demonstrate that neonates discriminate between infant- and adult-directed speech, attending more to and showing greater hemodynamic responses to the former than to the latter in bilateral frontal regions.

Exciting recent findings (May et al., under review), in which NIRS was used to record monolingual neonates' neural responses during exposure to auditory-only, low-pass filtered sentences in forward and backward native (or familiar) English, and non-native (or unfamiliar) Tagalog, showed that similar channels are activated for forward but not to backward speech in these infants. Moreover, there was no difference in lateralization observed for the two languages, with both eliciting bilateral hemodynamic functions. These findings suggest that similar regions are used to process native and non-native speech in monolingual neonates. However, infants in this study also showed no significant difference in response to forward and backward English conditions, a finding that contrasts with previous results obtained using unfiltered speech (Peña et al., 2003). The authors posit that the focused prosodic cues available in the low-pass filtered speech may have been driving the bilateral patterns of activation observed, as well as the atypical results concerning forward and backward English. Notably, the same stimuli were utilized in a previous study, which showed that bilingual exposure in utero resulted in bilingual neonates distinguishing between English and Tagalog (Byers-Heinlein et al., 2010).

Overall, these results add support to the view that left-lateralized language processing mechanisms are in place at birth. Of course, further research is needed to clarify the somewhat mixed neurophysiological evidence for differences in sensitivity to native compared with non-native speech processing in this age group (for a theoretical review of the NIRS left-lateralized speech literature see Minagawa-Kawai et al., 2011b). In particular, and in light of the comparison between speech and non-speech auditory stimuli with comparable complexity, the increased hemodynamic activity demonstrated to be specific to speech is compelling. Nonetheless, these data have not simplified theoretical debates about the degree to which nature and nurture come into play differentially in early language development. Rather, they have served to push the focal age for this debate ever earlier in development. Because of the paucity 
of neonatal (much less prenatal) data, we instead will focus on a well established postnatal phenomenon - categorical perception of speech sounds - to highlight how behavioral evidence has demonstrated that environment shapes biology strongly and quickly in the first year of life.

\section{BEHAVIORAL AND NEUROPHYSIOLOGICAL EVIDENCE OF NATIVE LANGUAGE SENSITIVITY IN NEONATES}

Given short exposure to the unfiltered speech signal, newborns demonstrate an impressive ability to process their native language. In spite of this limited experience, neonates can distinguish native speech from other complex, non-speech auditory stimuli when these are controlled for spectral and temporal factors (Vouloumanos and Werker, 2007). Recent research using NIRS has uncovered evidence of the neural mechanisms underlying these behavioral findings. Specifically, NIRS data demonstrate that the cortical areas utilized during speech processing are distinct compared with other auditory stimuli (for a review see Lloyd-Fox et al., 2010). For example, Kotilahti et al. (2010) compared music and speech processing in neonates. Overall, although no significant activation over baseline was observed in the right hemisphere for music or for speech, the auditory stimuli elicited bilateral activation, with increased responses in left relative to right temporal cortex during speech relative to music.

Apart from speech/non-speech distinctions, in utero experience with speech appears to be sufficient for newborns to differentiate between familiar speakers and stories in the context of native speech. For example, behavioral evidence clearly demonstrates that, within the first day of life, infants prefer their mother's voice over that of another woman (Decasper and Fifer, 1980). In addition, newborns can discriminate between a familiar (i.e., familiarized given prenatal exposure) and a novel story in their native language (Garnicka, 1977; Stern et al., 1983; Albin and Echols, 1996). They also discriminate between their own and another language from a different language family at birth. This recognition of familiar, native speech also extends to neurophysiological responses elicited from a single participant using electroencephalography (EEG). Radicevic et al. (2008) tested a single infant at 24 and 75 days of age. The child was read a story by the mother in her native tongue before and after birth (from 27 gestational weeks to 1 week before birth, then after birth for 7 days). Following birth, the infant was tested on several conditions: familiar (mother's) voice/familiar content, unfamiliar voice/familiar content, unfamiliar voice/familiar content in a non-native language. At 24 days, the familiar voice and content elicited a different delta rhythm from the non-native content in an unfamiliar voice, which resembled the resting state. In addition, at both ages, the unfamiliar language and voice elicited a response similar to rest. Moreover, 75-day measurements revealed similar delta and theta rhythms for both familiar and unfamiliar content regardless of language type or speaker familiarity.

\section{BEHAVIORAL EVIDENCE OF PERCEPTUAL TUNING AND IMPLICATIONS FOR NEURAL CORRELATES}

Perceptual tuning is a complex developmental phenomenon that has been the sole topic of substantial review papers (SebastianGalles, 2002; Werker and Tees, 2005). Here we focus specifically on the interplay between language environment and the neural mechanisms that support this tuning process. We will briefly discuss behavioral evidence for language-specific perceptual tuning, and follow up with a more detailed discussion of those neurophysiological data that can inform us about the neural sensitivities present during infancy that may underlie this.

Language tuning is the narrowing of perception of speech sounds over the first year of life, from an initially broad ability to distinguish many minimally contrastive phonemes to an increasingly specialized capacity to distinguish (for the most part) only those phonemes relevant to one's ambient language (Eimas et al., 1971; Jusczyk et al., 1977; Werker and Tees, 1983, 1999; Werker and Lalonde, 1988; Polka and Werker, 1994; Jusczyk, 1997). For the purpose of this discussion, the term "perceptual tuning" will be used synonymously with "perceptual narrowing" and "perceptual reorganization” (Best, 1994). As we have already seen, infants demonstrate tremendous skill in processing the speech stream from an early age, and a substantial behavioral literature has demonstrated that perceptual tuning to native speech occurs relatively early in development. Specifically, between 10 and 12 months of age infants with monolingual language profiles become more adept at discriminating native compared with non-native phonemic contrasts (Werker and Tees, 1984). In addition, Kuhl et al. (2006) demonstrated that monolingual English-exposed and monolingual Japanese-exposed 6- to 8-month-old infants were able to make the English/r/and/1/distinction, but by 10-12 months of age, only the monolingual English-exposed infants continued to differentiate these phonemes.

These data are further supported by event-related potential (ERP) data from 4- to 5-month-olds demonstrating different mismatch negativity signatures during exposure to pseudowords with a stress pattern common to the native language versus one uncommon to that language (Friederici et al., 2007). Moreover, another ERP study found infant responses indicative of both native and non-native consonant contrast discrimination in 7-month-olds, whereas 11-month-olds did not demonstrate such sensitivity contrasts (Rivera-Gaxiola et al., 2005). However, when analyses were based on a different parsing of ERP components, 11-month-old infants appeared to still be sensitive to both types of contrast. These conflicting results (though given differing methods of data analysis) suggest that differences in native compared with non-native consonant contrasts may not be as robust as once thought, thus requiring further investigation, particularly using ERP methods. In addition to conflicting results on consonant contrasts, behavioral findings have suggested a somewhat earlier timeline (e.g., 6-8 months) for vowel discrimination (Kuhl et al., 1992; Polka and Werker, 1994). Thus, one way to conceptualize perceptual narrowing is to view experience with a native language as sharpening the boundaries between native contrasts (Aslin and Pisoni, 1980; Kuhl et al., 2001; Polka et al., 2001).

Furthermore, recent work by Narayan et al. (2010) has uncovered evidence that some phoneme contrasts are not available "pre-tuning," while others (even non-native) remain available after tuning should (arguably) have ended (i.e., after 8 months). Specifically, these researchers found that the non-native/na/-/ $\mathrm{na}$

${ }^{1}$ The pre-tuning label is reflective of the traditional behavioral literature's timeline for language tuning in monolingual infants (i.e., before 6 months of age). 
contrast was not perceived by English 4- to 12-month-olds, nor was it perceived by Filipino 6- to 8-month-olds, for whom it was a native contrast. However, older Filipino 10- to 12-month-olds were able to make the distinction. This perceptual pattern is unusual, as non-native contrasts are typically perceived by pre-tuned infants of any language background (Trehub, 1976; Werker and Tees, 1984; Polka et al., 2001). This account highlights the intuitively appealing point that the perceptual tuning process is mediated by the relative difficulty or ease of speech signal processing (termed "acoustic salience" by Narayan et al. (2010). Given the behavioral evidence for perceptual tuning to speech over the first year of life, it is reasonable to assume that the neural mechanism(s) responsible for this process may be undergoing a concurrent and pronounced period of development. This could be considered the key critical, or sensitive, period.

\section{POSSIBLE NEURAL MECHANISMS FOR LANGUAGE TUNING IN INFANCY}

Needless to say, language development researchers have long debated the timing and degree of any so-called critical period (Johnson, 2001, 2005; Werker and Tees, 2005; Armstrong et al., 2006; Thomas and Johnson, 2008). The following summary of the critical/sensitive/optimal outcome period literature highlights how different perspectives on the issue may inform our understanding of the possible neural mechanisms underlying language tuning.

A critical period has been described as a fixed time range during which an organism's neural processing and behavior can be influenced by external environmental input (e.g., Werker and Tees, 2005). In contrast, a sensitive or optimal period (we will use the term sensitive period) is conceptualized as having a variable offset that depends on the organism's experience and learning (Knudsen, 1999), making the system adaptable to changing environmental inputs for a more flexible length of time. Importantly, Werker and Tees (2005) have reviewed evidence suggesting that different aspects of language (e.g., syntax, phonology, morphology, semantics) may each have their own critical period, or at least develop in a(n) "interrelated" or "nested" set of critical periods. Specifically, as infants gain more experience with language, they become (incrementally) more aware of progressively complex components and of variability in the speech signal.

Behavioral evidence of sequential mastery of different aspects of language has generated several theories about the plasticity of neural mechanisms in early development. Neural plasticity is a state of functional changes within the dynamic, iterative process of neural development. Although the current literature has yet to tease apart the functional significance of the increase in synaptogenesis and subsequent pruning of those synapses during infancy, some have suggested a link between peaks and subsequent elimination of synapses co-occurring with more mature function (Webb et al., 2001). While there are certainly dynamic and plastic aspects to the normally developing brain, the definition of plasticity we will use here focuses on the connections that are selectively retained post-pruning because they are used to process input (Stiles, 2000).

Theories applicable to language learning must therefore address plasticity and at least somewhat account for the influence of early language experience on the process. Trainor (2005) has proposed two possible explanations for the particularly strong influence of environment on neural development during infancy. The first, the genetically mediated account, posits that neural substrates' capacity to change and adapt in response to environmental inputs (such as those that that drive language tuning), is solely dependent upon maturation. Thus, infants would be expected to lose neural plasticity by a certain age regardless of environment, a position congruent with a "critical period" account of language tuning. Trainor's (2005) second explanation, the experientially mediated account, holds that experience facilitates brain organization by reducing neural plasticity as connections become more functionally specified. This theory is very similar to the neural commitment hypothesis (Kuhl et al., 2005b; Kuhl and Rivera-Gaxiola, 2008), which asserts that language environment shapes the creation of infants' neural connections (i.e., neural commitment). In particular, a language profile is thought to define the parameters of a "mental filter." Input that does not get through this filter is less effectively processed. Both of these theories are congruent with a sensitive, rather than critical, period perspective on language tuning, because experience (rather than time-based maturation) controls the reduction in neural plasticity.

Thomas and Johnson (2008) have reviewed several theories of the mechanisms underlying such period, and at least two of these may have direct relevance to understanding the neural basis for language tuning. First, their mechanism of termination of plasticity regulates the rate of synaptic pruning, such that pruning increases with the biological maturation of brain regions and as they become specialized to perform specific functions, such as sensory, motor, or cognitive processing. As with the genetically mediated account discussed by Trainor (2005), this perspective implies a biologically programmed (rather than experience-based) process, and therefore would support a critical period view of language tuning. One could argue that the initially robust ability of neonates to discriminate speech-sound contrasts from a variety of different specific languages, followed by the marked reduction in this ability months later, could be explained by a termination of plasticity or genetically mediated account. However, as some non-native contrasts remain available after the language tuning period ends (e.g., Narayan et al., 2010), and given that bilingually exposed infants have shown a relatively extended period of sensitivity to contrasts (Werker and Byers-Heinlein, 2008), it is difficult to completely reconcile the language tuning process with these strictly maturation-based accounts.

Thomas and Johnson (2008) self-termination of learning account relies instead on a mechanism in which learning itself is thought to create neural changes that reduce plasticity. Similar to the previously discussed experientially mediated account (see Trainor, 2005) and the neural commitment hypothesis (Kuhl et al., 2005b), this view holds that responses of particular brain regions emerge largely from their functional and anatomical connections with other areas; in other words, their specialization is ultimately activity-dependent. As such, these experience-based explanations are consistent with a sensitive period view, in which the narrowing of categorical perception of phonemes that occurs during language tuning could be explained as a function of the infant's exposure (or lack thereof) to those phonemes.

Experience-based explanations also complement an "acoustic salience" account of language tuning (Narayan et al., 2010), in which differential mastery for phoneme contrasts (with more salient ones being mastered first) begins to equalize as an infant 
gains experience with the less salient ones over time. These could also explain why sensitivity to some rarely encountered non-native phonemes, or native language non-speech sounds used to communicate expressions (e.g., clicks used to convey exasperation or pity by an English-speaker), remain when others are lost (Best et al., 1988). Finally, experience-based accounts could reveal why neonates with bilingual language profiles in utero show equal preference for both languages (Byers-Heinlein et al., 2010), whereas their monolingually exposed counterparts show preference only for their single native language (Mehler et al., 1988; Moon et al., 1993; Nazzi et al., 1998; Ramus et al., 2000).

Although the exact mechanisms underlying language tuning - whether critical or sensitive in nature - are still debated, the general concept of such a period has important practical implications, as studies have time and again demonstrated that the timing of infants' language exposure (and thus tuning) may predict future language abilities. For example, early perceptual sensitivity to simple non-native syllable contrasts at ages 6,14 , 15 , and 24 months has been shown to be correlated with subsequent language achievement, as established by the MacArthur Communicative Developmental Inventory performance on items such as phrases understood, words understood, and words produced (Tsao et al., 2004).

Kuhl et al. (2005b) have also reported that skilled early perception of native language phonemes can be a reliable predictor of later success in monolingual language development; in contrast, relatively sustained sensitivity to non-native language phoneme discrimination predicted a slower rate of language development. Kuhl et al. (2005b) argued that many markers of language development, including number of words produced, sentence complexity, and mean length of utterance, may be predicted based on an infant's early skill - or lack thereof - in discriminating native language phonemes. However, it is not clear from these behavioral data what might be driving the differences in the timing of language tuning and language development and its relation to subsequent language learning. We turn now to outcomes from neuroimaging and ERP studies that have attempted to identify possible neural mechanisms for this process.

\section{NEUROPHYSIOLOGICAL EVIDENCE OF LANGUAGE TUNING IN INFANCY}

Thus far, neurophysiological research on language learning in infants has revealed evidence of functional processing differences that may speak to the behavioral differences outlined in the previous section. In general, neurophysiological data demonstrate left-lateralized processing for language stimuli across the first 18 months of life. Notably, we will use the term lateralization to denote bilateral activity with significantly greater activity in the left compared with the right hemisphere. Most data on language processing fall into the category of lateralized, with bilateral activation being observed, though with one hemisphere (usually the left in the case of speech processing) showing significantly more activity than the other.

For example, fMRI data from typically developing, sleeping 2- to 3-month-olds demonstrated a significant left asymmetry (lateralization) for forward (but not backward) native speech (the infants were monolingually exposed), particularly in posterior superior temporal regions, whereas the same study showed bilateral responses in other regions (e.g., right dorsolateral prefrontal cortex; left angular gyrus) in otherwise matched 2- to 3-montholds (Dehaene-Lambertz et al., 2002). This lateralized response to forward, but not backward, speech was also observed in a NIRS study with sleeping neonates (Taga et al., 2007).

More recently, several NIRS studies have focused on testing awake and attentive infants to address the role of attentional state in infant language processing (Lloyd-Fox et al., 2010). For example, Taga and Asakawa (2007) exposed monolingual Japanese 2to 4-month-olds to unfamiliar native words and found bilateral temporal activation. Notably, the majority of stimulus presentation in this case was audiovisual, as the three-word stimuli were presented at different alternating and overlapping intervals with flashing $(4 \mathrm{~Hz})$ checkerboard. In contrast, Minagawa-Kawai et al. (2011a) exposed monolingual Japanese 4-month-olds to short sentences from auditory-only film dialogs or a speech database delivered by a male or female speaker. Analyses revealed left-lateralized hemodynamic activity for native compared with non-native speech conditions. Further evidence in support of the left-lateralization of native speech processing during infancy includes a series of NIRS studies from our own lab, demonstrating increased activation in the left relative to the right temporal regions in response to native language stimuli in older monolingual 6- to 9-month-old infants (Bortfeld et al., 2007, 2009).

Interestingly, a cross-sectional NIRS study using 3- to 28-montholds reported outcomes consistent with the idea of a reduction in plasticity for language tuning (Minagawa-Kawai et al., 2007). Awake monolingual infants were exposed to a set of four pseudowords that varied in terms of the duration of the last vowel. Specifically, two of the stimuli matched native language characteristics while the remaining two matched non-native characteristics. Total (i.e., a sum of oxygenated and deoxygenated) hemoglobin ${ }^{2}$ results showed significant left-lateralization in response to the native as compared to the non-native contrast in most of the older infants, whereas all of the youngest infants showed bilateral activation. Moreover, only older infants showed significant left-lateralization for the native contrasts. These results suggest that the emergence of lateralization to the left corresponds to the neural tuning to a specific language and corresponding reduction in plasticity, at least for monolingually exposed infants.

Discrepancies in the current literature may be at least partially explained by differences across experiments in methodologies, specific cortical measurement locations, details of the speech stimuli used (e.g., words, syllables, or sentences), attentional states (i.e., awake versus asleep), and whether the stimuli are auditory or audiovisual in nature (or mixed). For example, studies in which infants cycle in and out of sleep (e.g., DehaeneLambertz et al., 2002) appear to demonstrate different patterns of hemodynamic activity given these two different states of alertness. It would make sense that an infant's attentional state would impact their processing of speech and understanding the role that attention plays in neurophysiological measures will require addi-

${ }^{2}$ Total hemoglobin is an accepted but less utilized measure of hemodynamic activity because oxygenated hemoglobin generally provides the strongest signal to noise ratio (see Lloyd-Fox et al., 2010). 
tional research. It is also possible that different aspects of dynamic stimuli become more or less salient when presentation changes from uni- to multi-modal (Taga and Asakawa, 2007) and thus what is being measured may be different from studies in which stimulus presentation is consistently auditory or consistently audiovisual (Bortfeld et al., 2007, 2009; Minagawa-Kawai et al., 2011a). By the same token, studies that use auditory-only stimuli may differ from those that use audiovisual stimuli. Differences such as these across studies could be the source of variability in the degree of laterality observed. Nonetheless, at least for monolingually exposed infants, there seems to be converging evidence for relatively more involvement of the left than the right hemisphere during speech processing. However, this left-lateralized neurophysiological pattern of language processing may be influenced by experience with only language. Thus, we will next review the bilingual literature to examine the effect of additional language experience on the behavioral and neurophysiological aspects of language tuning in infancy.

\section{LANGUAGE TUNING IN BILINGUALLY EXPOSED INFANTS}

Theoretical accounts of the neural mechanisms involved in language tuning necessarily grapple with the interactive nature of biological and experiential factors. This dynamic relationship lies at the heart of data showing the fundamental impact of language environment on the language tuning process. Specifically studying the process of language tuning in bilingual infants, as compared to that in monolingual infants, is an important way to understand language tuning in light of a more diverse language profile (i.e., more complex experience). This holds the biological aspect of language constant between populations - in particular, when socioeconomic and other factors are likewise controlled, while contrasting the influence of language experience.

Of course, the degree to which bilingual language tuning differs from monolingual language tuning may depend upon the languages involved. The extant data suggest that comparison of bilingual and monolingual tuning timelines should be considered in the context of several factors, including the total number of contrastive phonemes within each language, the relative frequency of contrasts, the level of overlap between different categories across the languages, and, finally, the amount of exposure to each language profile (Burns et al., 2007; Sebastian-Galles and Bosch, 2009). These factors, in addition to choice of experimental paradigm, may account for the varied results currently available on the timing of language tuning in studies of monolingual versus bilingual infants (Curtin et al., 2011).

Current evidence suggests that early bilingual infants' language processing is similar to that of monolingual infants. Specifically in terms of differentiating native languages from one another, newborns with prenatal bilingual experience can discriminate (i.e., demonstrate equal preference for) their two rhythmically distinct languages at birth (Byers-Heinlein et al., 2010). Moreover, and relatively on par with the monolingual processing timeline, 4-month-old bilingual infants can discriminate either of two rhythmically similar languages from an unfamiliar language (with a different orientation response than monolinguals that is perhaps due to identifying the languages spoken before orienting; Bosch and Sebastian-Galles, 1997).
Thus, at least early in development, the pace of language tuning appears similar between infants with monolingual and bilingual language profiles.

Historically, results on bilingual language processing in infancy have appeared conflicted because bilingual infants have demonstrated language tuning at times on pace with, and at others lagging behind, their monolingual peers. Three studies in particular illustrate how monolingual and bilingual infants can retain similar language tuning timelines. First, using a habituation procedure, Burns et al. (2007) demonstrated that monolingual English and bilingual French-English 6- to 8-month-olds discriminated both English (i.e., /ba/and/pa/) and French voice onset time (VOT) contrasts (i.e., /ba/and/p $\mathrm{p}^{\mathrm{h}} \mathrm{a} /$ ) equally well. After 10 months, however, monolinguals only discriminated their native language contrast, while bilinguals remained able to make both distinctions. The authors note that these phonemic contrasts were high in frequency in both languages, and were likely unambiguous across languages, making this task relatively easy.

These results are congruent with findings using the head-turn preference paradigm to test monolingual English infants, monolingual Welsh infants, and English-Welsh bilingual infants, who demonstrated significantly longer looking times for familiar compared with unfamiliar VOTs (i.e., /ball/and/tall/) in their native language(s) at approximately 11 months of age (Vihman et al., 2007). Furthermore, Sundara et al. (2008) utilized an infant-controlled visual habituation procedure and exposed English monolinguals, French monolinguals, and French-English bilinguals to a contrast of the syllable/dae/, as the initial/d/phoneme differed in place of articulation for English and French (dental versus alveolar) and thus contrasted allophonically. Six- to 8-month-old infants from all three groups distinguished between these contrasts, while only the monolingual English and bilingual 10- to 12-month-olds were able to distinguish this contrast. These data suggest that, given highly frequent, similar phonemes with overlapping distributions across languages, bilingual infants remain on par with their monolingual counterparts. Thus, when overlap is coupled with high frequency in similar phonemes, overlap is unlikely to be a source of confusion or to cause delay in language tuning in bilingual infants.

However, other studies suggest language tuning may be delayed in bilingual infants compared with their monolingual counterparts. For example, Bosch and Sebastian-Galles (2003) used a familiarization/preference testing procedure to highlight differences in language tuning between monolingual and bilingual infants in the second half of the first year. Infants listened to disyllabic pseudowords with a stress pattern common to both languages, where the first vowel contrast was phonemic in Catalan, but not in Spanish (i.e., /e/versus/ $/$ /). Following exposure to the variable tokens of one pseudoword, they were then tested on their ability to discriminate between either two new tokens of the familiarized pseudoword or two new tokens of the alternate (novel) pseudoword. Analyses revealed that monolingual infants responded to the familiarized stimuli by 8 months of age, whereas bilingual infants demonstrated reliable familiarization-preference at 4 months of age and again at 12 months, but not at 8 months. The authors attributed the transitory failure in phonemic discrimination at 8 months to bilinguals' denser distribution of phonetic space than monolinguals, which 
could render recognition of salient distinctions while ignoring unimportant ones more difficult. Indeed, comparable behavioral delays in language tuning were observed in monolingual 8-monthold infants exposed to a crowded distribution of vowels (Sabourin et al., 2003) and stops (Conboy and Mills, 2006; Sundara et al., 2006, 2008; Fennell et al., 2007). As previously noted (Burns et al., 2007; Sundara et al., 2008), in addition to dense phonemic space, the frequency of the language contrasts used in the ambient language may also contribute to the relative timing of bilingual language tuning. Thus, it appears that both monolingual and bilingual infant populations cope with dense phonetic space by extending flexibility about this aspect of their language in the tuning process.

In order to further examine the roles of frequency and distribution of sounds on language tuning, Sebastian-Galles and Bosch (2009) tested 4-, 8-, and 12-month-old Spanish/Catalan bilingual infants with two vowel contrasts (i.e., /o-u/and/e-u/). As noted by the authors, the vowels chosen here were contrastive in both languages and were found in sparse phonemic space. Furthermore, the Spanish/u/is more infrequent than/o/, with the opposite distribution pattern found in Catalan. Using the/o-u/ contrast, a U-shaped pattern of discrimination was observed, where 4- and 12-month-olds were able to distinguish between the contrasts, while 8-month-olds were unable to perform this discrimination task, which was consistent with past data (Bosch and Sebastian-Galles, 2003). To further eliminate sources of ambiguity that may impact performance, Sebastian-Galles and Bosch (2009) tested 8-month-old bilingual infants using tokens uttered by fewer speakers, as well as tokens from a single speaker (i.e., reducing speaker-induced variability in the stimulus set). The data demonstrated that infants remained unable to perceive the/o-u/contrast, suggesting that variability introduced by multiple speakers does not affect performance. Given the more acoustically distinct/e-u/contrast, bilingual 8-month-olds (in addition to their monolingual peers) were able to discriminate the contrast. Thus, based on the properties of the/o-u/contrast, it seems unlikely that the statistical properties of phonemic contrasts were the only factors influencing language tuning. The authors concluded that other factors, such as the degree of lexical similarity of the languages within the profile, the type of contrast studied (i.e., vowel or consonant), the density of the phonemic environment, and "socio-indexical" factors (e.g., language-switching) are more likely to explain differences in performance for the/o-u/compared with the/e-u/contrasts. Clearly, the issue will require additional data before any concrete conclusions can be drawn. Taken together, results from these behavioral studies tell us that bilingual language tuning is a complex process likely impacted by many factors, some of which also affect monolingual infants, and others that are unique to this population (Curtin et al., 2011).

In addition to all this, the choice of experimental paradigm may also affect the outcomes of bilingual tuning studies. For example, a categorization task administered through a visual choice paradigm revealed a somewhat different developmental timeline for the tuning process than more traditional testing methods. The visual choice method is an adaptation of the anticipatory eye movement paradigm previously used as a change detection measure with speech stimuli (McMurray and Aslin,
2004). Albareda-Castellot et al. (2011) employed this approach in a categorization study that began with an attention-getting, visual reinforce. When the infant attended to the reinforcer, it moved behind a T-shaped occluder and a disyllabic word from one of two vowel categories was played three times (AlbaredaCastellot et al., 2011). Following initial occlusion, the reinforcer reemerged from the left or the right of the occluder, with the location predicted by the auditory stimulus. The reinforcer then reoriented the infants in preparation for the next trial. Based on the assumption that if they have already tuned to the auditory stimulus, infants will look in the direction predicted by that stimulus, the researchers analyzed the proportion of correctly anticipated place-of-emergence trials. They found that CatalanSpanish bilingual infants were appropriately tuned to a contrast common to both languages by the age of 8 months. They further observed that the bilingual infants kept pace with their monolingual peers for contrasts found in only one of the bilingual infants' languages (i.e., by 8 months). Thus, these results contrast with those from the habituation study by Bosch and SebastianGalles (2003). Given that the same contrasts were utilized in both studies, the conflict suggests that experimental design and choice of paradigm influenced an infants' demonstration of tuning. The possibility that the type of performance required in an experimental paradigm (rather than competence or ability) may account for the differential timing of monolingual and bilingual language tuning observed in previous studies highlights the need for replication with and across paradigms.

Furthermore, although Albareda-Castellot et al. (2011) results suggest that monolingually and bilingually exposed infants may experience a similar onset of native language tuning, it is not clear whether the degree of attainment (that is, competence) of language tuning is similar. It is also unclear precisely which differences in task demand may be driving the different outcomes between the familiarization-preference procedure and the visual choice method. One possibility lies in the "testing" portion of these two paradigms. For example, the familiarization-preference procedure can demonstrate discrimination between a single familiarized token $(\mathrm{F})$ and a novel token $(\mathrm{N})$ at test (F versus N; Quinn, 2002). Thus, using the familiarization-preference procedure, only one of the two stimuli at test ( $\mathrm{F}$ and not $\mathrm{N}$ ) are presented in a context or enriched manner (i.e., as a single repetition); this enrichment facilitates processing and has been termed intersensory facilitation, (Bahrick and Lickliter, 2000, 2002). In contrast, the visual choice method (or anticipatory eye movement paradigm) pairs each auditory stimulus with an associated spatial location. Given that intersensory facilitation is more apparent in tasks with redundant multi-modal information (i.e., information highlighting differences between stimuli), which could include perception of speech stimuli (Bahrick et al., 2010), it is thus possible that the visual choice paradigm allows infants to demonstrate more mature language tuning behavior due to its use of intersensory facilitation. This is in contrast to the familiarization-preference procedure, which uses an enriched presentation for only one of the two stimuli being tested, thereby limiting the observable results. Regardless, the discrepancies between these paradigms make it clear that future studies are needed to examine whether the timeline of bilingual language tuning may actually be shifted, 
or whether infant cognition may capitalize on different measurement procedures differently (Yoshida et al., 2009; Mattock et al., 2010; Albareda-Castellot et al., 2011).

In addition to the behavioral studies of bilingual language tuning in infancy, a handful of studies utilizing the ERP technique have examined neurophysiological correlates of language processing in bilingual infant populations. While these studies do not speak directly to differences in phonetic perception between monolinguals and bilinguals, they do provide insight into potential neurophysiological indicators of bilingualism in infancy. For example, a seminal study by Conboy and Mills (2006) examined 19- to 22-month-old English-Spanish bilinguals' neurophysiological responses to familiar and unfamiliar words in the children's dominant and non-dominant languages in the context of vocabulary size. These researchers found that the organization of the responses to words varied according to both individual and total vocabulary size, with higher producers producing a significant left-lateralized (P100) response in their dominant language. No such lateralization effect was observed in low producers. Furthermore, the researchers observed significantly different latencies (N200 versus N400 responses) for known compared with unknown words. Specifically, unknown words elicited a right lateralized response in the dominant language, while the non-dominant language demonstrated no such lateralization. Thus, these results demonstrate that language ability, as measured by vocabulary size, influences speed of processing and lateralization for processing the dominant and non-dominant languages.

Finally, Vihman et al. (2007) tested English monolingual and Welsh monolingual infants, as well as English-Welsh bilingual infants, all between 9 and 12 months of age, with ERP. Using familiar and unfamiliar word stimuli, these researchers observed the first neurophysiological indicator (i.e., in the form of an N2 response) of word form recognition at 10 months of age in English monolinguals, where behavioral data has shown such an effect only at 11 months of age. Furthermore, the ERP familiarity effect for English monolinguals vanished at 12 months in both paradigms, possibly indicating that the neurophysiological indicator of word form recognition is fundamentally different from the behavioral measure. Importantly, these authors noted that the Welsh monolingual infants showed no neurophysiological differences for the stimulus types, which may be due to unique qualities of the Welsh language (including mutation, reliance on later parts of words, and sociolinguistic factors). In contrast, 11-month-old English-Welsh bilingual infants showed the effect of familiarity in both languages and in both procedures, which may be due to the influence of English language learning on the Welsh language profile or to some of the other factors already mentioned. As with previously reviewed studies, elucidation of these findings will require additional research.

Ultimately, these data demonstrate that a bilingual language profile in infancy may result in different behavioral and neural consequences for language tuning in these (bilingual) infants compared with their monolingual peers. Numerous factors, including the languages involved in the bilingual language profile, the sociolinguistic environment, the level of overlap between the two languages, the total number of contrastive elements, and the frequency distribution of the input, all likely play a role in bilingual language tuning. Therefore, the take-home message about the time course of bilingual language tuning is, at present, unclear. However, the extant data certainly indicate that there are differences between bilingual and monolingual language profiles in the respective rates of behavioral evidence of tuning, patterns of related hemodynamic activity, and ultimate outcomes in language proficiency.

\section{LANGUAGE TUNING IN BILINGUALLY EXPOSED ADULTS}

Although data from bilingually exposed infants are sparse and not always consistent, substantial evidence from bilingual adults has shown that age of second language (L2) acquisition and L2 proficiency may dramatically influence language organization in the brain as well (Vingerhoets et al., 2003; Wartenbuger et al., 2003; Briellmann et al., 2004; Indefrey, 2006; Hull and Vaid, 2007). A structural MRI study involving monolinguals and early and late bilinguals found relatively increased gray matter density in the bilinguals in the inferior parietal lobe near the temporo-parietal junction, and significantly more so for early than late bilinguals (Mechelli et al., 2004). This suggests that the onset age of bilingualism and/or the length of L2 experience may alter the brain's actual structure.

In addition to anatomical differences in structure, numerous behavioral, and functional imaging studies have indicated differential language organization for early bilinguals relative to late bilinguals and monolinguals. For example, an fMRI study of multilingual adults with varying ages of L2 acquisition and different levels of L2 proficiency has demonstrated that late multilingual speakers consistently show left-lateralization for processing all languages, regardless of proficiency (Briellmann et al., 2004). However, the lone early multilingual in this study appeared to show bilateral activation patterns for all languages, including the (non-proficient) language the participant had acquired in adulthood (i.e., well outside the language tuning period in infancy). These outcomes would suggest that the neural substrates for language are different for bilinguals who acquired one (late bilinguals) versus multiple (early bilinguals) languages during early development.

Findings for differential neural bases for language processing in early and late bilinguals have also been demonstrated in languages that are not audiovisual in nature. For example, an fMRI study involving deaf and hearing sign language users showed that both hearing and deaf early American Sign Language (ASL)English bilinguals demonstrated bilateral activation during sign language processing (Neville et al., 1998). In contrast, late ASLEnglish bilinguals and ASL or English monolinguals displayed primarily left-lateralization of processing. These studies suggest that early exposure to multiple languages may result in recruitment of bilateral neural substrates - regardless of whether one of the languages is audiovisual and the other is not. Conversely, early exposure to a single language, again regardless of whether it is audiovisual or signed, may result in a left-lateralization for language. Finally, these outcomes suggest that the end result of language tuning may not only affect the languages present during that tuning process, but also any subsequently acquired languages. 
In terms of behavioral evidence for differences in brain organization for language between monolinguals and early and late bilinguals, two meta-analyses of the language laterality literature have provided support for this view. One meta-analysis specifically compared behavioral outcomes from monolinguals and early and late bilinguals; bilinguals were individually coded for age of L2 acquisition (early, late), and level of L2 proficiency (proficient, non-proficient), and only the first language of bilinguals that matched the language of the monolinguals was assessed (Hull and Vaid, 2006). The authors observed that late bilinguals and monolinguals showed left hemisphere language dominance, regardless of proficiency level, whereas early bilinguals demonstrated bilateral involvement for language. Hull and Vaid suggested that increased involvement of the right hemisphere in early bilinguals could be a consequence of a relatively early need to recruit right hemisphere pragmatic strategies, such as to facilitate understanding of when and with whom to use one language versus the other (Obler, 1981; Beeman and Chiarello, 1998; Boatman, 2004).

A second meta-analysis by Hull and Vaid (2007) focused specifically on disentangling laterality differences among early and late bilinguals for both their languages. The outcomes replicated Hull and Vaid (2007) by demonstrating reliable bilateral organization in early bilinguals and left-lateralization in late bilinguals for their first languages. In addition, lateralization within a particular bilingual subgroup overlapped across first and second languages; that is, both the L1 and L2 of early bilinguals were organized bilaterally, and both the L1 and L2 of late bilinguals were left hemisphere lateralized. Taken together, outcomes from these meta-analyses point to differences in brain organization associated with differences in the number of languages experienced during early development.

Based on these data, Hull and Vaid (2007) posited the anchoring hypothesis, which argues that early exposure to two (or more) languages necessitates recruitment of neural support bilaterally, whereas single language exposure requires only left hemisphere priority. Moreover, the early establishment of the functional language pattern "anchors" later-learned languages so that they display that same pattern (presumably because they rely on the same neural substrates that were specialized for early learned languages). As such, the anchoring hypothesis concerning bilingual language organization is consistent with experience-based accounts of perceptual tuning [e.g., the experientially mediated (Trainor, 2005) and neural commitment (Kuhl et al., 2005b; Kuhl and Rivera-Gaxiola, 2008) hypotheses].

Several PET and fMRI studies have demonstrated converging evidence for overlapping neural substrates supporting L1 and L2 in bilinguals, both at the single word (Chee et al., 1999; Illes et al., 1999; Klein et al., 1999; Hernandez et al., 2000) and continuous speech levels (Perani et al., 1996; Chee et al., 1999; Vingerhoets et al., 2003; Briellmann et al., 2004). Because the lateralization of languages has been shown to differ for early and late bilinguals, but the patterns of L1 and L2 processing are nonetheless overlapping within each bilingual subtype, these outcomes are consistent with the notion that the functional specificity of the neural bases of language remains static once they are established. Presumably, such functional specificity is set up during the sensitive (or critical) period for language tuning.
At present, the bulk of existing evidence on language organization in bilingual infants and adults suggests that the end result of language tuning not only affects the neural organization of languages acquired during tuning but also any that may be acquired later in life. Thus, it seems clear that language tuning may be different for bilinguals and monolinguals, and this warrants a revisiting of the theories that characterize the neural mechanisms thought to underlie plasticity and neural specificity for language function.

The experientially mediated hypothesis (Trainor, 2005) and the neural commitment hypothesis (Kuhl et al., 2005b; Kuhl and RiveraGaxiola, 2008) could explain differences in the timing of language tuning, as some behavioral evidence with bilingual infants suggests a delay in the completion of language tuning, presumably to accommodate additional language organization to ensure the integrity of processing for multiple language systems. This position would be consistent with behavioral and neurophysiological evidence for reliable differences in adult bilingual laterality depending on age of L2 acquisition. However, if bilingual infants complete tuning for both languages at the same pace as their monolingual peers complete tuning for a single language (as some behavioral studies have suggested), the diversity of bilingual input may not complicate organization after all. Although it remains for future studies with bilingual infants to resolve this debate, it is clearly difficult to reconcile the latter position with existing adult bilingual evidence or with the experientially mediated and neural commitment accounts of language tuning.

The self-termination of learning account would also suggest that different neural connections are being forged for bilingual compared to monolingual infants because of the differences in their language experiences. This theory would account for the differential language laterality observed in adult early bilinguals compared with monolingual late bilinguals. In addition, this explanation could account for differences in connection formation as a function of differences in age of L2 acquisition. However, the self-termination of learning could also be consistent with the idea of a similar tuning timeline for bilingual and monolingual populations, in which case the generation of neural connections for language tuning would be similar for single and multiple languages (although this would be at odds with the adult neurophysiological data). Therefore, in the context of the evidence presented here, a sensitive period account of language tuning appears to better explain the current data in the infant and adult bilingual literatures, with the caveat that a reexamination of the sensitive period explanation would be warranted if future research demonstrates that bilinguals and monolinguals have comparably timed schedules of language tuning.

\section{SENSORY DEPRIVATION (DEAFNESS) IN INFANTS AND CHILDREN}

While the infant bilingual literature can be useful in addressing how multiple language inputs may affect language tuning and its neural bases, the language profile of deaf children who have received cochlear implants (CI) may offer useful information about how the absence of early auditory language input impacts language tuning in the developing brain. That is, congenital 
deafness is a form of early sensory deprivation that can be later reversed by cochlear implantation surgery. What cannot be reversed, however, is the loss of early exposure, and this varies depending on the age of implantation. Given that tuning to native language phonemes in hearing infants requires auditory input and appears to develop during a period of high brain plasticity during infancy, the process of language tuning in children with CIs provides a unique opportunity to examine the boundary conditions for auditory plasticity and what, if any, language tuning takes place as a result.

Cochlear implants allow the recipient to experience auditory language for the first time, and this new sensory input ultimately results in neural reorganization to accommodate the perception of sound. Specifically, nearby brain areas are recruited to accommodate the new function of hearing (e.g., Lee et al., 2001; see also Desmond and Fiez, 1998; Recanzone, 2000, for related evidence from animal models). Importantly, CI research has demonstrated that cortical reorganization of the auditory cortex permits this region to adapt to the relatively limited frequency range produced by the CI, allowing for successful perception of even highly complex auditory stimuli (Shepherd et al., 1997). However, we are currently unaware whether the cortical reorganization during childhood cochlear implantation mimics that of infant language tuning.

The current literature focused on child CI users has identified several factors that influence the development of speech and language skills in a deaf child who is hearing through an implant. Unsurprisingly, evidence is accumulating that it is critical for the implantation to take place as early in development as possible (i.e., before 4 years of age). This allows the child to capitalize on the greater brain plasticity of that developmental period, thereby setting up the best conditions for normal auditory neural networks to emerge (Lee et al., 2001, 2007; Kang et al., 2004). Lee et al. (2001) used PET to show that by 7 years of age, cortical organization was significantly different from that of younger children, and this finding was corroborated by a converging paradigm (cortical auditory evoked potentials; Sharma et al., 2002). Later, Lee et al. (2007) and Kang et al. (2004) further narrowed the optimal window by establishing that post-implantation speech perception scores improved when implantation occurred before 4 years of age. More specifically, children who received a CI before the age of four performed best on sentence recognition tasks, whereas those implanted between 4 and 7 years of age showed a wide range of performance on this task; after age seven, implant recipients generally achieved low scores (Sharma et al., 2009).

However, as CI surgeries are performed on younger and younger patients we will be able to comment on how different durations of sensory deprivation affect language learning, and tuning. At least one recent behavioral study indicated that when testing infants with 1-2 months of initial CI use, infants that ranged between 4 and 10 months of age preferred their native language (Hebrew) compared with English (Kishon-Rabin et al., 2010). This timeline mirrors that seen for normal-hearing infants and corroborates the view that, with very early intervention, tuning may not be impacted (at least at the behavioral level).
Although the data from behavioral, PET, and EEG studies are helpful in providing evidence that the target period for normal language development via a CI following auditory deprivation ends by 4 years of age (Gordon et al., 2005; Dorman et al., 2007), it is generally quite difficult to obtain neurophysiological measurements from very young children using these techniques. Moreover, fMRI cannot be used in CI patients because the devices are incompatible with the scanner's magnetic field. However, the NIRS technique allows measurement of blood-oxygen level dependent changes in cortical activity, similar to fMRI, without interference with the implant. Indeed, NIRS evidence regarding language development has already been used to study the bilateral temporal responses of pediatric CI recipients to language.

Sevy et al. (2010) tested CI children (mean age 7.8 years, $n=7$ ) on the same day they received their implants, providing a unique opportunity to observe how the untrained auditory cortex responds to its first exposure to sound. The results revealed significant hemodynamic responses to speech stimuli in the majority of deaf children, and the volume of hemodynamic response was not significantly different from that of hearing controls (mean age 10.2 years, $n=9$ ). However, the majority of deaf children with a new CI showed a unilateral hemodynamic response to the auditory stimuli, and that response was most often ipsilateral to the location of the CI (typically in the right hemisphere). Once the children had at least 6 months of experience with their CI, responses to the auditory stimuli were predominantly bilateral and the unilateral responses that remained were most likely to be contralateral to the CI. These findings provide an initial glimpse into the boundaries for plasticity in congenitally deaf children whose deafness is subsequently ameliorated with a CI.

While this is the first study to use NIRS with this population, the Sevy et al. (2010) results demonstrate that NIRS can safely measure cortical responses in pediatric implant users, thereby establishing NIRS as a valuable tool for investigating the neural bases of language tuning and development in this special population. This application of NIRS promises to expand our understanding of the boundary conditions for development of normal auditory processing and of the likely neural mechanisms involved in perceptual tuning in general, and language tuning in particular. Thus, NIRS and other converging neurophysiological paradigms may further elucidate how sensory deprivation impacts auditory brain development in children (Neville and Bavelier, 2002; Kang et al., 2004; Giley et al., 2008).

\section{CONCLUSION AND SYNTHESIS}

Traditionally, researchers have discussed language tuning in terms of a critical, or sensitive, period (Werker and Tees, 2005). These terms identify language tuning as a phenomenon that is either constrained by biological maturation of systems important to language learning or as a period that capitalizes on the responsiveness of neural plasticity to language experience. In the context of language tuning, the present review highlights the importance of the differential role of experience (i.e., a child's language profile) in actively shaping the biological foundation for language best characterize the extant data. Three theories that characterize this aspect of the data particularly well are the self-termination of 
learning account (Thomas and Johnson, 2008), the experientially mediated account (Trainor, 2005), and the neural commitment hypothesis (Kuhl et al., 2005a).

Although these theoretical constructs offer valuable explanations of the processes that drive perceptual tuning in general, they are somewhat limited in terms of their ability to explain the range of behavioral and neurophysiological data on language tuning. Based on the synthesis of data presented here, it appears that language tuning may be comprised of staggered, nested components that may tune at different times (Werker and Tees, 2005). Thus, a more specific means of reconciling these data can be encompassed with a supplementary explanation, namely, the relative speed of tuning hypothesis. Specifically, the relative speed of tuning hypothesis predicts that, within the maturational window that clearly exists for native-like language learning, the more transparent, obvious elements of language may tune faster than more opaque, ambiguous elements of language. We anticipate that this hypothesis will be especially useful in accounting for conflicting data within the literature, as it allows researchers to break the tuning process down based on the specific factors that influence tuning rate as measured using behavioral and neurophysiological paradigms. In particular, this approach has the potential to account for the impact of the in utero language profile, the characteristics of both heterogeneous and homogenous language profiles during infancy and childhood, and the roles of age and sensory deprivation on language tuning. Our hypothesis gives rise to several predictions, some of which are supported by the current data and others that will require further investigation.

First, the relative speed of tuning hypothesis predicts that behavioral testing with transparent, relatively easy tasks should demonstrate evidence of tuning in younger populations than those with more complicated, opaque tasks. For example, use of a categorization paradigm may demonstrate behavioral evidence of tuning earlier than a discrimination paradigm, due to the relative difficulties of these tasks (Bosch and Sebastian-Galles, 2003; Albareda-Castellot et al., 2011). And this should also be the case for neurophysiological paradigms, where evidence of language tuning should be observed earlier given tasks that tap into elements of language that tune early themselves. For example, an experimental design contrasting native and non-native languages of a different rhythmic class should demonstrate neural indicators of language tuning earlier than a task contrasting native and non-native languages within the same rhythmic class.

A further prediction is that "robust," or cue-filled, sentence-level speech stimuli will elicit indicators of language tuning sooner than, for example, filtered speech (in which phonetic cues are removed) or contrast-level stimuli (in which only a fraction of the original speech stimulus is retained). This is borne out by recent neurophysiological data, in which infants showed initial neural evidence of tuning to word-level contrasts at 11 months (Minagawa-Kawai et al., 2007), while showing evidence of tuning to sentence-level stimuli at 4 months (Minagawa-Kawai et al., 2011a). Thus, evidence of language tuning - that is, a more left-lateralized hemodynamic response for native compared to non-native speech - was elicited in a younger group of infants given more robust stimuli. Our hypothesis also accounts for data from studies comparing infants' ability to discriminate phonemes from more or less populated phonemic space in which discrimination is better for phonemes from sparsely populated phonemic space relative to those from dense phonemic space (Burns et al., 2007; Sumner et al., 2008).

Some cues may be more robust than others given different language profiles. For example, ambiguity due to changes in speaker appears to be less influential to language tuning in bilingually exposed infant than do the relative frequency of phonetic contrasts across the ambient languages (Sebastian-Galles and Bosch, 2009). Since the relative speed of tuning hypothesis predicts that linguistic elements that are more robust and transparent will elicit earlier tuning than will weaker or more complex elements, different predictions emerge for bilingual relative to monolingual profiles. For example, where May et al. (under review) utilized speech stimuli that were essentially only prosodic in nature (i.e., low-pass filtered) and failed to obtain neural indicators of discrimination between native and non-native forms in monolingual neonates, bilingual neonates were able to distinguish between these same stimuli in a behavioral task (Byers-Heinlein et al., 2010). The relative speed of tuning hypothesis would predict such a difference given the different language profiles of the two groups of infants. Although unavailable from these studies, one can imagine that the full complement of data (e.g., monolingual and bilingual infants tested with the same stimuli using both behavioral and neurophysiological measures) would further support this outcome. The influence of these and other factors require further investigation to determine their relative impact on rate of language tuning.

Finally, the relative speed of tuning hypothesis can also account for data from populations deprived of sensory information, such as cochlear implant users. For these individuals, some of whom receive auditory input only after their system has undergone language tuning without it, we predict that tuning would occur more quickly for more robust aspects of the auditory signal that can be integrated with the greatest ease (e.g., phonemes that are clearly mapped to the visual signal, such as bilabial stops, or prosodic contours that easily map to, for example, vocal aperture or facial prosody). Again, such research will further inform the field.

In sum, given different language profiles, the relative speed of language tuning hypothesis provides a flexible way of framing the complex and sometimes contradictory behavioral and neurophysiological literature. Of course, this hypothesis is only one of several trying to account for the diverse data on language tuning. This review highlights the need for converging evidence from a variety of experimental designs, linguistic stimuli, and imaging modalities and neurophysiological methods to demonstrate reliable evidence of language tuning and to resolve some of the current inconsistencies across studies and language profiles. It is possible that evidence (both neural and behavioral) of language tuning will emerge from increased use of ecologically valid, sentence-level stimuli (as opposed to single syllable and pseudoword stimuli). Because the majority of neurophysiological data on early language development have been obtained from monolingually exposed infants, it will also be important for future studies to investigate the influences of different language profiles on the neural mechanisms that support language tuning. Nonetheless, the present review makes clear that the extant literature offers an important guide for future exploration of the basis of the language tuning process. 


\section{REFERENCES}

Albareda-Castellot, B., Pons, F., and Sebastián-Gallés, N. (2011). The acquisition of phonetic categories in bilingual infants: new data from an anticipatory eye movement paradigm. Dev. Sci. 14, 395-401.

Albin, D. D., and Echols, C. H. (1996). Stressed and word-final syllables in infant-directed speech. Infant Behav. Dev. 19, 401-418.

Armstrong, V. L., Brunet, P. M., He, C., Nishimura, M., Poole, H. L., and Spector, F. J. (2006). What is so critical?: a commentary on the reexamination of critical periods. Dev. Psychobiol. $48,326-331$

Aslin, R.N., and Pisoni,D. B. (1980)."Some developmental processes in speech perception," in Child Phonology, eds. G. H. Yeni-Komshian, J. F. Kavanagh, and C. A. Ferguson (New York, NY: Academic Press), 67-96.

Bahrick, L. E., and Lickliter, R. (2000). Intersensory redundancy guides attentional selectivity and perceptual learning in infancy. Dev. Psychol. 36, 190-201.

Bahrick, L. E., and Lickliter, R. (2002). "Intersensory redundancy guides early perceptual and cognitive development," in Advances in Child Development and Behavior, ed. R. Kail (New York, NY: Academic Press), 153-187.

Bahrick, L. E., Lickliter, R., Castellanos, I., and Vaillant-Molina, M. (2010). Increasing task difficulty enhances effects of intersensory redundancy: testing a new prediction of the Intersensory Redundancy Hypothesis. Dev. Sci. 13, 731-737.

Beeman, M., and Chiarello, C. (1998). Complementary left and right hemisphere language comprehension. Curr. Dir. Psychol. Sci. 7, 2-8.

Best, C. (1994). "The emergence of native-language phonological influences in infants: a perceptual assimilation model," in The Development of Speech Perception: The Transition from Speech Sounds to Spoken Words, eds J. C. Goodman and H. C. Nusbaum (Cambridge, MA: The MIT Press), 167-224.

Best, C., Mcroberts, G. W., and Sithole, N. M. (1988). Examination of perceptual reorganization for nonnative speech contrasts: Zulu click discrimination by English-speaking adults and infants. J. Exp. Psychol. Hum. Percept. Perform. $14,345-360$

Boatman, D. (2004). Cortical bases of speech perception: evidence from functional lesion studies. Cognition 92, 47-65.

Bortfeld, H., Fava, E., and Boas, D. A. (2009). Identifying cortical lateraliza- tion of speech processing in infants using near-infrared spectroscopy. Dev. Neuropsychol. 34, 52-65.

Bortfeld, H., Wruck, E., and Boas, D. A. (2007). Assessing infants' cortical response to speech using nearinfrared spectroscopy. NeuroImage 34, 407-415.

Bosch, L., and Sebastian-Galles, N. (1997). Native-language recognition abilities in 4-month-old infants from monolingual and bilingual environments. Cognition 65, 33-69.

Bosch, L., and Sebastian-Galles, N. (2003). Simultaneous bilingualism and the perception of a language specific vowel contrast in the first year of life. Lang. Speech 46, 217-244.

Briellmann, R. S., Saling, M. M., Connell, A. B., Waites, A. B., Abbott, D. F., and Jackson, G. D. (2004). A high-field functional MRI study of quadrilingual subjects. Brain Lang. 89, 531-542.

Burns, T. C., Yoshida, K. A., Hill, K., and Werker, J. F. (2007). The development of phonetic representation in bilingual and monolingual infants. Appl. Psycholinguist. 28, 455-474.

Byers-Heinlein, K., Burns, T. C., and Werker, J. F. (2010). The roots of bilingualism in newborns. Psychol. Sci. 21, 343-348.

Chee, M. W. L., Tan, E. W. L., and Thiel, T. (1999). Mandarin and english single word processing studied with functional magnetic resonance imaging. J. Neurosci. 19, 3050-3056.

Conboy, B. T., and Mills, D. L. (2006). Two languages, one developing brain: effects of vocabulary size on bilingual toddlers' event-related potentials to auditory words. Dev. Sci. 9, F1-F11.

Curtin, S., Byers-Heinlein, K., and Werker, J. F. (2011). Bilingual beginnings as a lens for theory development: PRIMIR in focus. J. Phon. doi: 10.1016/j. wocn.2010.12.002. [Epub ahead of print].

Decasper, A. J., and Fifer, W. P. (1980). Of human bonding: newborns prefer their mothers' voices. Nature 208, 1174-1176.

Decasper, A. J., Lecanuet, J. P., Busnel, M. C., Granier-Deferre, C., and Maugeais, R. (1994). Fetal reactions to recurrent maternal speech. Infant Behav. Dev. $17,159-164$.

Dehaene-Lambertz, G., Dehaene, S., and Hertz-Pannier, L. (2002). Functional neuroimaging of speech perception in infants. Science 298, 2013-2015.

Desmond, J. E., and Fiez, J. A. (1998). Neuroimaging studies of the cerebellum: language, learning and memory. Trends. Cogn. Sci. 2, 355-362.

Dorman, M. F., Gifford, R. H., Spahr, A. J., and Mckarns, S. A. (2007). The benefits of combining acoustic and electric stimulation for the recognition of speech, voice and melodies. Audiol. Neurootol. 13, 105-112.

Eimas, P. D., Siqueland, E. R., Jusczyk, P. W., and Vigorito, J. (1971). Speech perception in infants. Science 171 303-306.

Fennell, C. T., Byers-Heinlein, K., and Werker, J. F. (2007). Using speech sounds to guide word learning: the case of bilingual infants. Child Dev. 78, 1510-1525.

Friederici, A., Friederich, M., and Christophe, A. (2007). Brain responses in 4-month-old infants are already language specific. Curr. Biol. 17, 1208-1211.

Garnicka, O.K. (1977). "Some prosodic and paralinguistic features of speech to young children," in Talking to Children: Language Input and Acquisition, eds C. E. Snow and C. A. Ferguson (Cambridge: Cambridge University Press), 63-88.

Gervain, J., Macagno, F., Cogoi, S., Pena, M., and Mehler, J. (2008). The neonate brain detects speech structure Proc. Natl. Acad. Sci. U.S.A. 105, 14222-14227.

Giley, P. M., Sharma, A., and Dorman, M. F. (2008). Cortical organization in children with cochlear implants. Brain Res. 1239, 56-65.

Gordon, K. A., Tanaka, S., and Papsin, B. C. (2005). Atypical cortical responses underlie poor speech perception in children using cochlear implants. NeuroReport 16, 2041-2046.

Hepper, P., and Shabidullah, B. (1994). Development of fetal hearing. Arch. Dis. Child Fetal Neonatal Ed. 71 , F81-F87.

Hernandez, A. E., Martinez, A., and Kohnert, K. (2000). In search of the language switch: an fMRI study of picture naming in Spanish-English bilinguals. Brain Lang. 73, 421-431.

Hull, R., and Vaid, J. (2006). Laterality and language experience. Laterality $11,436-464$.

Hull, R., and Vaid, J. (2007). Bilingual language lateralization: a metaanalytic tale of two hemispheres. Neuropsychologia 45, 1987-2008.

Illes, J., Francis, W. S., Desmond, J. E., Gabrieli, J. D. E., Glover, G. H. Poldrack, R., Lee, C. J., and Wagner, A. D. (1999). Convergent cortical representation of semantic processing in bilinguals. Brain Lang. 70, 347-363.

Indefrey, P. (2006). "A meta-analysis of hemodynamic studies on first and second language processing: which suggested differences can we trust and what do they mean?," in The Cognitive Neuroscience of Second Language Acquisition, eds M. Gullberg and P.
Indefrey (Malden, MA: Blackwell), 279-304.

Jardri, R., Pins, D., Houfflin-Debarge, V., Chaffiotte, C., Rocourt, N., Pruvo, J.P., Steinling, M., Delion, P., and Thomas, P. (2008). Fetal cortical activation to sound at 33 weeks of gestation: a functional MRI study. NeuroImage 42, 10-18.

Johnson, M. H. (2001). Functional brain development in humans. Nat. Rev. Neurosci. 2, 475-483.

Johnson, M. H. (2005). Sensitive periods in functional brain development: problems and prospects. Dev. Psychobiol. 46, 287-292.

Jusczyk, P. W. (1997). The Discovery of Spoken Language. Cambridge, MA: MIT Press.

Jusczyk, P. W., Rosner, B. S., Cutting, J. E., Foard, C. F., and Smith, L. B. (1977) Categorical perception of nonspeech sounds by 2 -month-old infants. Percept. Psychophys. 21, 50-54.

Kang, E., Lee, D. S., Kang, H., Lee, J. S., Oh, S., Lee, M. C., and Kim, C. S. (2004). Neural changes associated with speech learning in deaf children following cochlear implantation. NeuroImage 22, 1173-1181.

Kishon-Rabin,L.,Harel,M.,Hildesheimer, M., and Segal, O. (2010). Listening preference for the native langauge compared to an unfamiliar language in hearing and hearing-impaired infants after cochlear implantation. Otol. Neurotol. 31, 1275-1280.

Kisilevsky, B. S., Hains, S. M. J., Jacquet, A. Y., Grainer-Deferre, C., and Lecanuet, J. P. (2004). Maturation of fetal responses to music. Dev. Sci. 7 , 550-559.

Klein, D., Milner, B., Zatorre, R., Zhao, V., and Nikelski, J. (1999). Cerebral organization in bilinguals: a PET study of Chinese-English verb generation. NeuroReport 10, 4841-4846.

Knudsen, E. I. (1999). Mechanisms of experience-dependent plasticity in the auditory localization pathway of the barn owl. J. Comput. Physiol. A $185,305-321$

Kotilahti, K., Nissila, I., Nissila, T., Lipiainen, L., Noponen, T., Merilainen, P., Huotilainen, M., and Fellman, V. (2010). Hemodynamic responses to speech and music in newborn infants. Hum. Brain Mapp. 31, 595-603.

Kuhl, P. K., Conboy, B. T., Coffey-Corina, S., Padden, D., and Nelson, T. (2005a). Early speech perception and later language development: implications for the "critical period." Lang. Learn. Dev. $1,237-264$.

Kuhl, P. K., Conboy, B. T., Padden, D. Nelson, T., and Pruitt, J. (2005b). Early speech perception and later language development: implications for the 
“critical period." Lang. Learn. Dev. 1, 237-264.

Kuhl, P. K., and Rivera-Gaxiola, M. (2008). Neural substrates of language acquisition. Annu. Rev. Neurosci. 31, 511-534.

Kuhl, P. K., Stevens, E. B., Hayashi, A., Deguchi, T., Kiritani, S., and Iverson, P. (2006). Infants show a facilitation effect for native language phonetic perception between 6 and 12 months. Dev. Sci. 9, F13-F21.

Kuhl, P. K., Tsao, F. M., Liu, H. M., Zhang, Y., and Deboer, B. (2001). Language/ culture/mind/brain: progress at the margins between disciplines. Annu. N. Y. Acad. Sci. 935, 136-174.

Kuhl, P. K., Williams, K. A., Lacerda, F., Stevens, K. N., and Lindblom, B. (1992). Linguistic experience alters phonetic perception in infants by 6 months of age. Science 255, 606-608.

Lecanuet, J. P., Fifer, W. P., Krasnegor, N., and Smotherman, W. P. (1995). Fetal development: a psychobiological perspective. Hillsdale, NJ: Lawrence Erlbaum Association.

Lee, H. J., Truy, E., Mamou, G., SappeyMarinier, D., and Giraud,A.-L. (2007). Visual speech circuits in profound acquired deafness: a possible role for latent multimodal connectivity. Brain 130, 2929-2941.

Lee, S. D., Lee, J. S., Oh, S. H., Kim, S. K., Kim, J. W., Chung, J. K., Lee, M. C., and Kim, C. S. (2001). Deafness: cross-modal plasticity and cochlear implants. Nature 409, 149-150.

Lloyd-Fox, S., Blasi, A., and Elwell, C. E. (2010). Illuminating the developing brain: the past, present and future of functional near infrared spectroscopy. Neurosci. Behav. Rev. 34, 269-284.

Mattock, K., Polka, L., Rvachew, S., and Krehm, M. (2010). The first steps in word learning are easier when the shoes fit: comparing monolingual and bilingual infants. Dev. Sci. 13, 229-243.

McMurray, B., and Aslin, R. N. (2004). Anticipatory eye movements reveal infants' auditory and visual categories. Infancy 6, 203-229.

Mechelli, A., Crinion, J. T., Noppeney, U., O'doherty, J., Ashburner, J., Frackowiak, R. S., and Price, C. J. (2004). Neurolinguistics: structural plasticity in the bilingual brain. Nature $431,757$.

Mehler, J., Bertoncini, J., and Barriere, M. (1978). Infant recognition of mother's voice. Perception 7, 491-497.

Mehler, J., Jusczyk, P. W., Lambertz, G., Halsted, G., Bertoncini, J., and AmielTison, C. (1988). A precursor of language acquisition in young infants. Cognition 29, 143-178.

Minagawa-Kawai, Y., Van Der Lely, H., Ramus, F., Sato, Y., Mazuka, R., and Dupoux, E. (2011a). Optical brain imaging reveals general auditory and language-specific processing in early infant development. Cereb. Cortex 21, 254-261.

Minagawa-Kawai, Y., Cristia, A., and Dupoux, E. (2011b). Cerebral lateralization and early speech acquisition: a developmental scenario. Dev. Cogn. Neurosci. 1, 217-232.

Minagawa-Kawai, Y., Mori, K., Naoi, N., and Kojima, S. (2007). Neural attunement processes in infants during the acquisition of a language-specific phonemic contrast. J. Neurosci. 27, 315-321.

Moon, C., Panneton-Cooper, R., and Fifer, W.P. (1993). Two-day olds prefer their native language. Infant Behav. Dev. 16, 495-500.

Morokuma, S., Fukushima, K., Kawai, N., Tomonaga, M., Satoh, S., and Nakano, H. (2004). Fetal habituation correlates with functional brain development. Behav. Brain Res. 153, 459-463.

Narayan, C. R., Werker, J. F., and Beddor, P. S. (2010). The interaction between acoustic salience and language experience in developmental speech perception: evidence from nasal place discrimination. Dev. Sci. 13, 407-420.

Nazzi, T., Bertoncini, J., and Mehler, J. (1998). Language discrimination by newborns: toward an understanding of the role of rhythm. J. Exp. Psychol. Hum. Percept. Perform. 24, 756-766.

Neville, H., and Bavelier, D. (2002). Human brain plasticity: evidence from sensory deprivation and altered language experience. Prog. Brain Res. 138, 177-188.

Neville, H. J., Bavelier, D., Corina, D., Rauschecker, J., Karni, A., Lalwani, A., Braun, A., Clark, V., Jezzard, P., and Turner, R. (1998). “Cerebral organization for language in deaf and hearing subjects: biological constraints and effects of experience," in Proceedings of the National Academy of Sciences (Washington, DC: The National Academy of Sciences), 95, 922-929.

Obler, L. K. (1981). "Right hemisphere participation in second language acquisition," in Individual Differences and Universals in Language Learning Aptitude, ed. K. Diller (Rowley, MA: Newbury Press), 53-64.

Peña, M., Maki, A., Kovačić, D., DehaeneLambertz, G., Koizumi, H., Bouquet, F., and Mehler, J. (2003). Sounds and silence: an optical topography study of language recognition at birth. Proc. Natl. Acad. Sci. U.S.A. 100, 11702-11705.

Perani, D., Dehaene, S., Grassi, F., Cohen, L., Cappa, S. F., Dupoux, D., Fazio, F., and Mehler, J. (1996). Brain processing of native and foreign languages. NeuroReport 7, 2439-2444.
Polka, L., Colantontio, C., and Sundara, M. (2001). A cross-language comparison of/d/-/th/perception: evidence for a new developmental pattern. J. Acoust. Soc. Am. 109, 2190-2201.

Polka, L., and Werker, J. F. (1994). Developmental changes in perception of non-native vowel contrasts. J. Exp. Psychol. Hum. Percept. Perform. 20, 421-435.

Quinn, P. C. (2002). "Early categorization: a new synthesis," in Blackwell Handbook of Childhood Cognitive Development, ed. U. Goswami (Malden, MA: Blackwell), 84-101.

Radicevic, Z., Vujovic, M., Jelicic, L., and Sovilj, M. (2008). Comparative findings of voice and speech: language processing at an early ontogenetic age in quantitative EEG mapping. Exp. Brain. Res. 184, 529-532.

Ramus, F., Hauser, M. D., Miller, C., Morris, D., and Mehler, J. (2000). Language discrimination by human newborns and by cotton-top tamarin monkeys. Science 288, 349-351.

Recanzone, G.H. (2000)."Cerebral cortical plasticity: perception and skill acquisition," in The New Cognitive Neurosciences, 2nd ed., ed. M. S. Gazzaniga, (Cambridge,MA:MIT Press),237-247.

Rivera-Gaxiola, M., Silva-Pereyra, J., and Kuhl, P. K. (2005). Brain potentials to native and non-native contrast in 7and 11-month-old american infants. Dev. Sci. 8, 162-172.

Sabourin, L., Werker, J. F., Bosch, L., and Sebastián-Gállès, N. (2003). Perceiving vowels in a tight vowel space: evidence from monolingual infants. J. Cogn. Dev. Available at: http://aixl.uottawa. ca/ Isabour3/publicat.htm

Saito, Y., Aoyama, S., Kondo, T., Fukumoto, R., Konishi, N., Nakamura, K., Kobayashi, M., and Toshima, T. (2007). Frontal cerebral blood flow change associated with infant-directed speech. Arch. Dis. Child Fetal Neonatal Ed. 92, F113-F116.

Sebastian-Galles, N. (2002). Comment on Werker and Tees (1984). Crosslanguage speech perception: evidence for perceptual reorganisation during the first year of life. Infant Behav. Dev. 25, 144-146.

Sebastian-Galles, N., and Bosch, L. (2009). Developmental shift in the discrimination of vowel contrasts in bilingual infants: is the distributional account all there is to it? Dev. Sci. 12, 874-887.

Sevy, A., Bortfeld, H., Huppert, T., Beauchamp, M. S., Tonini, R., and Oghalai, J. (2010). Neuroimaging with near-infrared spectroscopy demonstrates speech-evoked activity in the auditory cortex of deaf children following cochlear implantation. Hear. Res. 270, 39-47.
Sharma, A., Dorman, N. F., and Spahr, A. J. (2002). A sensitive period for the development of the central auditory system in children with cochlear implants: implication for age of implantation. Ear Hear. 23, 532-539.

Sharma, A., Nash, A. A., and Dorman, M. (2009). Cortical development, plasticity and re-organization in children with cochlear implants. J. Commun. Disord. 42, 272-279.

Shepherd, R. K., Hartmann, R., Heid, S., Hardie, N., and Klinke, R. (1997). The central auditory system and auditory deprivation: experience with cochlear implants in the congenitally deaf. Acta Otolaryngol. 117, 28-33.

Stern, D. N., Spieker, S., Barnett, R. K., and Mackain, K. (1983). The prosody of maternal speech: infant age and context related changes. J. Child Lang. 10, 1-15.

Stiles, J. (2000). Neural plasticity and cognitive development. Dev. Neuropsychol. $18,237-272$.

Sumner, P., Anderson, E. J., Sylvester, R., Haynes, J. D., and Rees, G. (2008). Combined orientation and colour information in human V1 for both $\mathrm{L}-\mathrm{M}$ and S-cone chromatic axes. NeuroImage 39, 814-824.

Sundara, M., Polka, L., and Genesee, F. (2006). Language-experience facilitates discrimination of/d-/in monolingual and bilingual acquisition of English. Cognition 100, 369-388.

Sundara, M., Polka, L., and Molnar, M. (2008). Development of coronal stop perception: bilingual infants keep pace with their monolingual peers. Cognition 108, 232-242.

Taga, G., and Asakawa, K. (2007) Selectivity and localization of cortical response to auditory and visual stimulation in awake infants aged 2 to 4 months. NeuroImage 36, 1246-1252.

Taga, G., Homae, F., and Watanabe, H. (2007). Effects of source-detector distance of near infrared spectroscopy on the measurement of the cortical hemodynamic response in infants. NeuroImage 38, 452-460.

Thomas, M. S. C., and Johnson, M. H. (2008). New advances in understanding sensitive periods in brain development. Curr. Dir. Psychol. Sci. $17,1-5$.

Trainor, L. J. (2005). Are there critical periods for musical development? Dev. Psychol. 46, 262-278.

Trehub, S. E. (1976). The discrimination of foreign speech contrasts by infants and adults. Child Dev. 47, 466-472.

Tsao, F. M., Liu, H. M., and Kuhl, P. K. (2004). Speech perception in infancy predicts language development in the second year of life: a longitudinal study. Child Dev. 75, 1067-1084. 
Vihman, M. M., Thierry, G., Lum, J. A., Keren-Portnoy, T., and Martin, P. (2007). Onset of word form recognition in English, Welsh, and English-Welsh bilingual infants. Appl. Psycholinguist. 28, 475-493.

Vingerhoets, G., Van Borsel, J., Tesink, C., Van Den Noort, M., Deblaere, K., Seurinck, R., Vandemaele, P., and Achten, E. (2003). Multilingualism: an fMRI study. NeuroImage 20, 2181-2196.

Vouloumanos, A., and Werker, J. F. (2007). Listening to language at birth: evidence for a bias for speech in neonates. Dev. Sci. 10, 159-164.

Wartenbuger, I., Heekeren, H. R., Abutalebi, J., Cappa, S. F., Villringer, A., and Perani, D. (2003). Early setting of grammatical processing in the bilingual brain. Neuron 37, 159-170.
Webb, S. J., Monk, C. S., and Nelson, C. A. (2001). Mechanisms of postnatal neurobiological development: implications for human development. Dev. Neuropsychol. 19, 147-171.

Werker, J. F., and Byers-Heinlein, K. (2008). Bilingualism in infancy: first steps in perception and comprehension. Trends Cogn. Sci. 12, 144-151.

Werker, J. F., and Lalonde, C. E. (1988). Cross-language speech perception: initial capabilities and developmental change. Dev. Psychobiol. 24, 672-683.

Werker, J. F., and Tees, R. C. (1983). Developmental changes across childhood in the perception of non-native speech sounds. Can. J. Psychol. 37, 278-286.

Werker, J. F., and Tees, R. C. (1984). Crosslanguage speech perception: evidence for perceptual reorganization during the first year of life. Infant Behav. Dev. 7, 49-63.

Werker, J. F., and Tees, R. C. (1999). "Experimential influences on infant speech processing: toward a new synthesis," in Annual Review of Psychology, ed. J. T. Spence (Palo Alto, CA: Annual Reviews), 509-535.

Werker, J. F., and Tees, R. C. (2005).Speech perception as a window for understanding plasticity and commitment in language systems of the brain. Dev. Psychobiol. 46, 233-251.

Yoshida, K. A., Fennell, C. T., Swingley, D., and Werker, J. F. (2009). Fourteenmonth-old infants learn similarsounding words. Dev. Sci. 12, 412-418.

Conflict of Interest Statement: The authors declare that the research was conducted in the absence of any commercial or financial relationships that could be construed as a potential conflict of interest.

Received: 15 January 2011; accepted: 12 July 2011; published online: 01 August 2011. Citation: Fava E, Hull R and Bortfeld $H$ (2011) Linking behavioral and neurophysiological indicators of perceptual tuning to language. Front. Psychology 2:174. doi: 10.3389/fpsyg.2011.00174

This article was submitted to Frontiers in Language Sciences, a specialty of Frontiers in Psychology.

Copyright (C) 2011 Fava, Hull and Bortfeld. This is an open-access article subject to a non-exclusive license between the authors and Frontiers Media SA, which permits use, distribution and reproduction in other forums, provided the original authors and source are credited and other Frontiers conditions are complied with. 